\title{
YouTube as a Source of Patient Information for Bipolar Disorder: A Content- Quality and Optimization Analysis
}

\author{
Tomasz Szmuda ${ }^{1, \#,}$, Weronika Żydowicz ${ }^{2, \#, *}$, Shan Ali ${ }^{2, \#,}$ Karolina Fedorow ${ }^{2, \#}$ and Paweł Słoniewski ${ }^{2}$ \\ ${ }^{1}$ Neurosurgery Department, Medical University of Gdansk, Poland \\ ${ }^{2}$ Student's Scientific Circle of Neurology and Neurosurgery, Neurosurgery Department, Medical University of Gdansk, Poland \\ \#Equally contributed
}

*Corresponding author: Weronika Żydowicz, MD, MPH, Student's Scientific Circle of Neurology and Neurosurgery, Neurosurgery Department, Medical University of Gdansk, Poland, Tel: +4866477915; Fax: 0048583493330; E-mail: veronikazydowicz@wp.pl

Received: 14 Apr, 2021 | Accepted: 07 May, 2021 | Published: 15 May, 2021

Citation: Szmuda T, Żydowicz W, Ali S, Fedorow K, Słoniewski P (2021) YouTube as a Source of Patient Information for Bipolar Disorder: A Content-Quality and Optimization Analysis. J Psychiatry Ment Health 6(2): dx.doi.org/10.16966/2474-7769.141

Copyright: (C) 2021 Szmuda T, et al. This is an open-access article distributed under the terms of the Creative Commons Attribution License, which permits unrestricted use, distribution, and reproduction in any medium, provided the original author and source are credited.

Abstract

Objectives: YouTube is currently the second most popular website in the world and it is often used by patients to access health information online. We aimed to evaluate the content-quality of YouTube videos relating to bipolar disorder.

Methods: We chose the first 30 videos for four different search phrases: "bipolar disorder," "bipolar disorder treatment," "bipolar disorder symptoms" and "manic depression". Video contents were evaluated by two independent final-year medical students using the validated DISCERN instrument. Qualitative data, quantitative data and the upload source was recorded for analysis.

Results: Out of the total 120 videos, 80 videos met our inclusion criteria and were evaluated. The mean DISCERN score was 63.5 (out of 75 possible points). This indicates that the quality of YouTube videos on bipolar disorder is excellent. Reliability between the two raters was excellent (intraclass correlation coefficient $=0.96)$. Nearly all videos had symptoms of the disorder $(100 \%)$ and the impact of the disorder on daily life $(98.8 \%)$. Videos were mostly uploaded by educational channels $(61.3 \%)$ and hospitals (16.3\%). Videos that had a doctor speaker had a significantly higher average daily views, comments and a video power index $(P<0.05)$.

Conclusions: The quality of YouTube videos on bipolar disorder is good. We have included a list of the top-quality videos in our paper as they may be used by patients and physicians as a reference to find the most reliable videos for patient education. Having a doctor speaker in a video optimizes a video for higher audience engagement.

Keywords: Bipolar disorder; Internet; Online; Psychiatry; YouTube

\section{Background}

Approximately 4.6 billion people are active internet users; this translates to $60 \%$ of the world population [1]. YouTube is the most common platform for video viewing worldwide [1]. The general public uses YouTube primarily as a source of entertainment but it has also expanded as a medium for education. $90 \%$ of people aged 18 to 24 years trust medical information shared by others online [2]. YouTube's large volume of information and accessibility attracts doctors, medical students, patients, and their families to use it as a source of medical information. Healthcare-related videos published on YouTube are often not reliable and may contain misinformation $[3,4]$. While there have been studies regarding the role of YouTube videos disseminating psycho education for dementia, schizophrenia and narcolepsy they have been of poor or limited quality, there are currently no studies on the quality of videos for bipolar disorder (BD) [5-7].
$\mathrm{BD}$ is a mental disorder characterized by shifts in mood and ranging from depression to mania, with periods of euthymia where the patient does not have mood disturbances. The euthymic phase is the best time to treat and provide psycho-educational advice. Bipolar I (BP1) is defined by prolonged periods of mania and depression, often requiring hospitalization. Bipolar II (BP2) is defined by cycles of hypomanic episodes and depression. It affects $1 \%$ of the population worldwide and the median age of onset is 25 years [8]. It is found equally among men and women and it prevails in all social classes, races and ethnicities [8].

The percentage of patients with $\mathrm{BD}$ who use the internet is the same as the general public at about $81 \%$ [9]. Most patients with BD use the internet to find information about their disorder but also consult two other sources such as medical professionals, books and physician handouts [9]. Only $21 \%$ of these patients read or participate in support groups, chats, or forums for $\mathrm{BD}[10]$ and 
most do not discuss Internet information concerning $\mathrm{BD}$ with their physician [11].

Individuals with $\mathrm{BD}$ consistently report social stigma as one of the greatest challenges of living with the condition and thus anonymously search for medical information online (rather than seeking professional help) [12]. For this reason, BD is often misdiagnosed or can go undiagnosed for up to 10 years which perpetuates the suffering from the disease [13]. However, several of the other factors are involved and the delayed diagnosis of $\mathrm{BD}$ including first-degree relatives, high stress, trauma, illicit drug usage and most importantly, depressive disorders/emotional lability like neuroticism.

We aimed to assess the current quality and the reliability of You Tube videos on $\mathrm{BD}$ using a comprehensive search strategy. We also sought to determine what optimizes a video for audience engagement.

\section{Materials and Methods}

\section{Search strategy}

We searched YouTube on November 11, 2019 using the four key phrases; "bipolar disorder", "bipolar disorder treatment", "bipolar disorder symptoms", and "manic depression". We felt that these search terms offered a sufficient view of BD videos because they are relative synonyms to BD. We intentionally chose these phrases since after being given a diagnosis of "bipolar disorder" we anticipated that a patient would probably use the same search term or a slight variation of it on YouTube.

\section{Data collection}

Previous research shows that $90 \%$ of search engine users only look at the first 3 pages of searched content. Thus, the first 30 results of each search were viewed and evaluated under the chosen criteria and results were recorded [14].

\section{Inclusion and exclusion criteria}

Out of 120 videos, we evaluated a total of 80 videos related to BD. Any duplicates, advertisements, music videos, videos in another language or videos clearly not related to BD (e.g., music videos) were excluded.

\section{Variables extracted}

We used the Google Chrome extension "VidIQ Vision for YouTube" to retrieve quantitative information from each video. This included: total number of views, total number of comments, duration, video description word count, video description link count, referrers, likes, dislikes, and video tags and time since upload. Information about the host channel was also recorded: uploader name, average daily views, average daily subscribers, subscribers, and tags.

Qualitative information included: impact of the disorder on everyday life, symptoms of $\mathrm{BD}$, personal story/vignette of person, the difference between BP1 and BP2, results of treatment, discussion of prognosis, animations, diagrams, pathomechanisms explained, a doctor speaker and/or a patient experience. We recorded the video upload source and categorized it into one of the following categories: physician, hospital, patient, educational or other.

\section{Scoring system}

Videos were evaluated using the validated DISCERN instrument, by two medical students in the final clinical years of their studies who each had five years of experience using the DISCERN instrument. The DISCERN instrument is a sixteen-point questionnaire designed to allow laypeople to judge the reliability and quality of health information as seen in table $1[15,16]$. The first fifteen questions are scored on a scale from 1-5 depending on if they meet the given criteria. A score of 1 means the video did not fulfill the criteria, partial fulfillment of criteria can be scored a 2-4 depending on the independent judgment of the scorer, and a 5 is a definite yes. The total minimum score is 16 and the maximum score is 80 points.

The last question is a summary of the previous 15 DISCERN questions and determines whether the publication can be used as an appropriate source of information. An overall rating of 2 or below indicates the publication is of "poor" quality with many shortcomings and is not useful or appropriate. A score of 3 indicates a "fair" quality of publication that requires additional sources of information due to some limitations. A score of 4 or above indicates "good" quality meaning the publication is useful and appropriate as a source of information about treatment choices $[15,16]$.

The DISCERN score may also be interpreted as a total out of 75 using only the first 15 questions. A score of 63 to 75 indicates an "excellent" score, 51 to 62 indicates a "good" score, 39 to 50 indicates a "fair" score, 27-38 indicates a "poor" score and 16 to 26 indicates a "very poor" score $[17,18]$.

\section{Video optimization}

The optimization analysis method in this study is similar to studies

Table 1: The 16 question DISCERN Instrument.

\begin{tabular}{|c|c|c|c|c|c|c|}
\hline $\mathrm{Nr}$ & Question & \multicolumn{5}{|c|}{ Rating } \\
\hline 1 & Are the aims clear? & 1 & 2 & 3 & 4 & 5 \\
\hline 2 & Does it achieve its aims? & 1 & 2 & 3 & 4 & 5 \\
\hline 3 & Is it relevant? & 1 & 2 & 3 & 4 & 5 \\
\hline 4 & $\begin{array}{l}\text { Is it clear what sources of information } \\
\text { were used to compile the publication } \\
\text { (other than the author or producer)? }\end{array}$ & 1 & 2 & 3 & 4 & 5 \\
\hline 5 & $\begin{array}{l}\text { Is it clear when the information used } \\
\text { or reported in the publication was } \\
\text { produced? }\end{array}$ & 1 & 2 & 3 & 4 & 5 \\
\hline 6 & Is it balanced and unbiased? & 1 & 2 & 3 & 4 & 5 \\
\hline 7 & $\begin{array}{l}\text { Does it provide details of additional } \\
\text { sources of support and information? }\end{array}$ & 1 & 2 & 3 & 4 & 5 \\
\hline 8 & Does it refer to areas of uncertainty? & 1 & 2 & 3 & 4 & 5 \\
\hline 9 & $\begin{array}{l}\text { Does it describe how each treatment } \\
\text { works? }\end{array}$ & 1 & 2 & 3 & 4 & 5 \\
\hline 10 & $\begin{array}{l}\text { Does it describe the benefits of each } \\
\text { treatment? }\end{array}$ & 1 & 2 & 3 & 4 & 5 \\
\hline 11 & $\begin{array}{l}\text { Does it describe the risks of each } \\
\text { treatment? }\end{array}$ & 1 & 2 & 3 & 4 & 5 \\
\hline 12 & $\begin{array}{l}\text { Does it describe what would happen if } \\
\text { no treatment is used? }\end{array}$ & 1 & 2 & 3 & 4 & 5 \\
\hline 13 & $\begin{array}{l}\text { Does it describe how the treatment } \\
\text { choices affect overall quality of life? }\end{array}$ & 1 & 2 & 3 & 4 & 5 \\
\hline 14 & $\begin{array}{l}\text { Is it clear that there may be more than } \\
1 \text { possible treatment choice? }\end{array}$ & 1 & 2 & 3 & 4 & 5 \\
\hline 15 & $\begin{array}{l}\text { Does it provide support for shared } \\
\text { decision making? }\end{array}$ & 1 & 2 & 3 & 4 & 5 \\
\hline 16 & $\begin{array}{l}\text { Based on the answers to all of these } \\
\text { questions, rate the overall quality } \\
\text { of the publication as a source of } \\
\text { information about treatment choices }\end{array}$ & 1 & 2 & 3 & 4 & 5 \\
\hline
\end{tabular}


previously published $[4,7,19-21]$. The average daily views (total views/ days since upload), the like ratio ((likes/likes+dislikes) $\left.{ }^{*} 100\right)$ and the video power index (VPI) $\left((\text { like } 100 /(\text { like+dislike }))^{\star}(\right.$ views/day $\left.) / 100\right)$ were used to assess the audience engagement with a video. Of note, standardized cut-off scores do not exist for the VPI since it depends on the number of likes and views and this is highly variable depending on how popular a video is. However, we have provided the mean VPI in the results section so that a reader may use it for reference (to see if a VPI value is higher or lower than the mean).

Videos were grouped based on their qualitative content (e.g., if a video included the treatment of BP disorder). These video groups were analyzed against their average daily views, like ratio, VPI and number of comments.

\section{Statistical methods}

The normality was checked and in cases where the data were normally distributed, the Mann-Whitney $U$ test was used to find differences between categorical variables. The intraclass correlation coefficient was used to ascertain inter-rater agreement. $\mathrm{P}<0.05$ was deemed significant. Google Sheets (Google LLC, Mountain View, California, USA) was used for illustrations. Past (Hammer and Harper, Øyvind Hammer, Natural History Museum, University of Oslo) was used for statistical analysis.

\section{Results}

\section{Video contents}

A total of 80 unique videos were included in our content analysis. Figure 1 shows the qualitative content of videos about $\mathrm{BD}$. Almost all videos covered the symptoms (100\%) and the impact (99\%) of BD on daily life. Most videos included the treatment $(60 \%)$, the prognosis (54\%), a personal vignette (54\%) and a patient experience concerning $\mathrm{BD}(51 \%)$. However, most videos did not have a doctor speaking $(48 \%)$, animation (28\%), BD pathomechanisms $(25 \%)$, a diagram (23\%), and the differences between BD1 \& BD2 (31\%).

\section{Video upload source}

Figure 2 illustrates the source of the videos uploaded. The majority of the videos were uploaded by an educational channel 61.3\% (49 videos). The remainder of the videos was uploaded by a hospital $16.3 \%$ ( 13 videos), a physician $11.3 \%$ (9 videos), a patient $8.8 \%$ ( 7 videos) and $2.5 \%$ ( 2 videos) were uploaded by other sources.

\section{Video statistics}

The following represent the mean quantitative aspects of the videos overall: duration 1034.1 (47-7573) seconds, view count 323059 (10613500000), comments 702.3 (0-5960), likes 6011.4 (0-61000), dislikes 153.8 (0-1683), average daily views 41 (0-630), like ratio 93.9 (50-100), video referrers $71.7(0-527)$ and days since upload 1459.325 (1254294).

The following represent the mean channel popularity of the videos: subscribers 1713569 (3-21700000), daily views 6824841.4 (49.8$486900000)$ and daily subscribers 39832.6 (0.1-1550000).

\section{Video quality evaluation}

The mean DISCERN score of the two raters for DISCERN was 63.4 \pm 11.3 (38-80) (out of 80 possible points). The first rater and second rater had a mean DISCERN score of $63.5 \pm 11.3$ (39-80, and $63.3 \pm$ 11.3 (38-80), respectively. The intraclass correlation coefficient for the absolute agreement between the two raters was 0.996 ( $95 \%$ Confidence Interval of 0.9938 to 0.9975$)$; this is regarded as "excellent" reliability according to Cicchetti and Koo [22-24].

The mean sum of the first 15 items of DISCERN was 59.3 (out of 75 total points). This is considered a "good" quality of information (62-51 points) [17].

The mean DISCERN score between the two raters for question 16 was $4.1(2-5)$. The mean score for each rater was $4.1(2-5)$ and $4.1(2-5)$, respectively. This may be regarded as a "good" quality of information that is useful for patients making treatment choices. Specifically, 59 videos $(73.75 \%)$ scored a 4 or above indicating a good quality of the publication.

The mean total DISCERN scores differed in regards to the uploader: patient videos scored the lowest at 47.64 while physician video uploads scored the highest at 68.11. Hospital videos scored 62.23 and educational channel videos scored 64.93 .

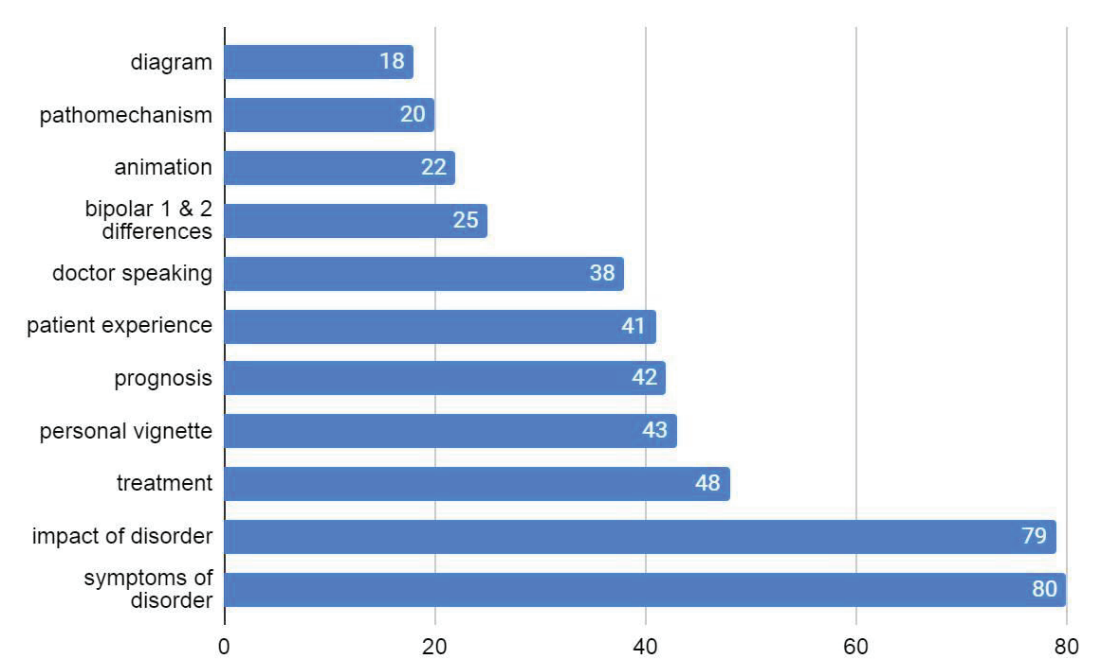

Figure 1: Videos contents for bipolar disorder. 


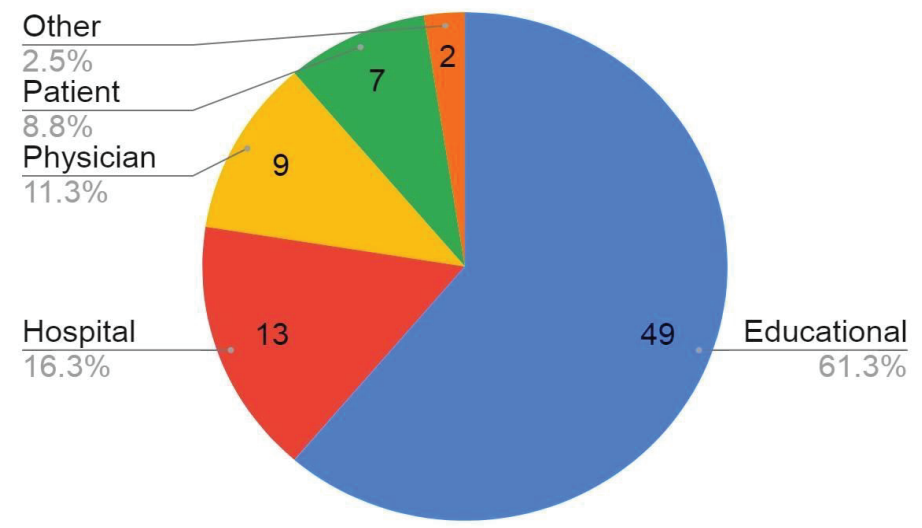

Figure 2: Source of video uploads on bipolar disorder.

Figure 3 illustrates the mean score for each of the 16 questions of DISCERN. Questions 1 to 3 scored the highest ratings overall. They pose essential questions in regards to the qualitative video content: are the aims clear, does it achieve its aims, and is it relevant. Questions 4 to 8 all scored above 4 , this is considered a "good" score. These five questions rate the publication based on the publication's clarity of sources of information, clarity of when the information was reported or used, bias, the inclusion of additional sources, and uncertainty. The lowest scores were for questions 9 to 15. These questions are focused explicitly on treatment or lack thereof: how it works, what are the benefits, what are the risks, how they affect the quality of life, and the variety of treatments.

\section{Video quality correlations}

Table 2 shows video quality in relation to the video content. Videos had a greater DISCERN score when they included: the results of the treatment $(\mathrm{P}=0.0001)$, the prognosis $(\mathrm{P}=0.0001)$, the pathomechanisms $(\mathrm{P}=0.0003)$, a doctor speaker $(\mathrm{P}=0.0004)$ and diagrams $(\mathrm{P}=0.0098)$.

\section{Optimization analysis}

Table 3 shows that audience engagement concerning the video content. Videos that contained an animation $(\mathrm{P}=0.034)$ or a doctor speaker $(\mathrm{P}=0.0006)$ had significantly higher average daily views and a higher VPI. There was a statistically significant relationship with a doctor speaker $(\mathrm{P}=0.0005)$ in the video and a higher number of comments. There were no statistically significant findings in regards to the like ratio.

\section{Top-quality YouTube videos}

Table 4 shows the highest-scoring YouTube videos. The three topscoring videos were tied with 80 points and the following 3 were tied with 79 points. The mean DISCERN score of the top six videos is 79.5 $(99.375 \%)$, which means patients may view them as a trusted source of health content. Half of the videos were uploaded by an educational channel, two were uploaded by hospitals and one was uploaded by a physician.

All these videos included the following five features: symptoms of $\mathrm{BD}$, the impact of the disorder on everyday life, results of treatment, discussion of prognosis and a doctor speaker. In general, these videos were 4 times longer in duration and (on average 4028 seconds in comparison to the average of all videos 1034) covered an entire gamut of information.

\section{Discussion}

\section{Quality analysis}

The overall quality and reliability of the bipolar videos were of good quality. Thus, patients may gain basic knowledge about BD on YouTube. Healthcare professionals have a duty to provide their patients with reliable medical information. Since it is impractical to peer-review all videos and other information on the internet, we have listed the top 6 highest quality videos on BD so that physicians may share reliable educational YouTube content to their patients over online communications (e.g., e-mail, social media, hospital websites). While there is research on other neuropsychiatric disorders on the internet and on YouTube, our paper is the first to cover an in-depth analysis of the quality and reliability of BD.

Our study shows that YouTube contains adequate medical information concerning BD. However, several factors were often omitted, these included: the risks of each treatment, the consequences of treatment, the benefits of each treatment, how treatment would affect the overall quality of life, how each treatment works, and the possibility of more than one treatment option. Future video creators may focus on these aspects as they are frequently omitted. Including this information would not only increase the quality of the videos, but also the popularity. We suggest that medical institutions tasked with publishing medical YouTube videos should consider our recommendations and use the DISCERN tool as a checklist for what should be included in their videos to provide a valid information package. We encourage hospitals to publish high-quality and informative videos on $\mathrm{BD}$ and other medical topics.

\section{Optimization analysis}

Prior studies found that patients do not engage with the highest quality educational videos [25]. Our study has comparable findings. Thus, it is critical for content creators (i.e., hospital staff and medical professionals) to understand what makes a video attractive and engaging to the viewer. This way, the most informative videos may be given attention instead of being ignored.

Our results show that videos containing an animation garnered the highest average daily views and VPI. This demonstrates that visual aids may make videos more appealing to viewers. The presence of a doctor speaker resulted in more comments, average daily views and VPI. This indicates that patients are more inclined to watch and interact with 


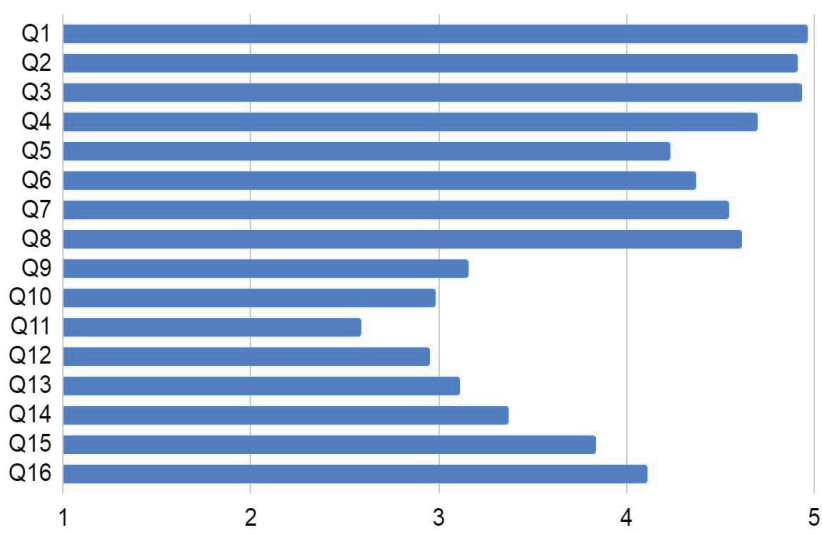

Figure 3: The mean score of the two raters for the 16 DISCERN instrument questions.

Abbreviation: Q: question

Table 2: Statistically significant relationships between the DISCERN score and selected qualitative video content.

\begin{tabular}{|c|c|c|}
\hline & With information & Without information \\
\hline & \multicolumn{2}{|c|}{ DISCERN } \\
\hline \multicolumn{3}{|l|}{ Results of treatment } \\
\hline Mean & 70.563 & 52.578 \\
\hline 95\% Confidence interval & 68.583 to 72.542 & 49.927 to 55.230 \\
\hline Sample size & 48 & 32 \\
\hline$P$ value & \multicolumn{2}{|c|}{0.0001} \\
\hline \multicolumn{3}{|l|}{ Prognosis } \\
\hline Mean & 70.726 & 55.237 \\
\hline $95 \%$ Confidence interval & 68.462 to 72.990 & 52.218 to 58.255 \\
\hline Sample size & 42 & 38 \\
\hline$P$ value & \multicolumn{2}{|c|}{0.0001} \\
\hline \multicolumn{3}{|l|}{ Diagrams } \\
\hline Mean & 69.028 & 61.726 \\
\hline 95\% Confidence interval & 63.601 to 74.455 & 58.947 to 64.504 \\
\hline Sample size & 18 & 62 \\
\hline$P$ value & \multicolumn{2}{|c|}{0.0098} \\
\hline \multicolumn{3}{|l|}{ Pathomechanism } \\
\hline Mean & 70.750 & 60.908 \\
\hline 95\% Confidence interval & 66.577 to 74.923 & 58.073 to 63.744 \\
\hline Sample size & 20 & 60 \\
\hline$P$ value & \multicolumn{2}{|c|}{0.0003} \\
\hline \multicolumn{3}{|l|}{ Doctor speaker } \\
\hline Mean & 67.395 & 59.726 \\
\hline 95\% Confidence interval & 63.870 to 70.919 & 56.409 to 63.043 \\
\hline Sample size & 38 & 42 \\
\hline$P$ value & \multicolumn{2}{|c|}{0.0004} \\
\hline
\end{tabular}

Table 3: Statistically significant relationships between the average daily views, comments and the video power index against qualitative video content.

\begin{tabular}{|c|c|c|}
\hline & With information & Without information \\
\hline & \multicolumn{2}{|c|}{ Average daily views } \\
\hline \multicolumn{3}{|l|}{ Animation } \\
\hline Mean & 940.6 & 408.74 \\
\hline 95\% Confidence interval & -123.84 to 2005.00 & 150.66 to 666.82 \\
\hline Sample size & 22 & 58 \\
\hline$P$ value & \multicolumn{2}{|c|}{0.034} \\
\hline \multicolumn{3}{|l|}{ Doctor speaker } \\
\hline Mean & 218.29 & 859.64 \\
\hline 95\% Confidence interval & 34.57 to 402.01 & 242.71 to 1476.60 \\
\hline Sample size & 38 & 42 \\
\hline \multirow[t]{2}{*}{$P$ value } & \multicolumn{2}{|c|}{0.0006} \\
\hline & \multicolumn{2}{|c|}{ Comments } \\
\hline \multicolumn{3}{|l|}{ Doctor speaker } \\
\hline Mean & 263.49 & 1096.2 \\
\hline 95\% Confidence interval & 98.331 to 428.64 & 591.16 to 1601.20 \\
\hline Sample size & 35 & 35 \\
\hline \multirow[t]{2}{*}{$P$ value } & \multicolumn{2}{|c|}{0.0005} \\
\hline & \multicolumn{2}{|c|}{ Video power index } \\
\hline \multicolumn{3}{|l|}{ Animation } \\
\hline Mean & 914.33 & 402.77 \\
\hline 95\% Confidence interval & -126.14 to 1954.80 & 151.29 to 652.26 \\
\hline Sample size & 22 & 57 \\
\hline$P$ value & \multicolumn{2}{|c|}{0.0489} \\
\hline \multicolumn{3}{|l|}{ Doctor speaking } \\
\hline Mean & 217.8 & 833.69 \\
\hline 95\% Confidence interval & 32.061 to 403.53 & 234.71 to 1432.70 \\
\hline Sample size & 37 & 42 \\
\hline$P$ value & \multicolumn{2}{|c|}{0.0008} \\
\hline
\end{tabular}


Table 4: The top six highest quality bipolar disorder videos based on the DISCERN criteria.

\begin{tabular}{|c|c|c|c|}
\hline DISCERN & Title & Uploader & YouTube ID \\
\hline 80 & Bipolar disorder: treatment and preventing relapse-Dr Patrick McKeon & Aware & LCeLKT2SFQo \\
\hline 80 & Treatment of bipolar disorder & NAMI SGV & Q2KNgzVYQZo \\
\hline 80 & Treatment choices: options for bipolar disorder & DBS Alliance & gzgi9Sr7twY \\
\hline 79 & Treating bipolar disorder & Paul Merritt & wpYIrJ_ild0 \\
\hline 79 & Understanding and treating bipolar disorder & Free CE & WfkgvBHPOYQ \\
\hline 79 & Understanding bipolar depression & Stanford Hospital & b_ZxPOubM6A \\
\hline
\end{tabular}

videos uploaded by credible medical professionals. This is a reassuring finding to know that patients appreciate or may be trying to reproduce the classical doctor-patient interaction on a digital interface. The presence of a patient experience was another determinant of high popularity indicating that patients value information coming from a relatable source.

However, we highlight that a direct cause-effect relationship between content (e.g., a doctor speaker) and audience engagement metric (e.g., average daily views) may not be able to be established since the viewer discovers the content of the video only after it is clicked. For example, unless the title or thumbnail indicates the content of the video (e.g., "Doctor X talks about Bipolar Disorder" or a thumbnail of a person in a white coat) the viewer would not know that there is a doctor speaker before clicking it. Of all the audience engagement metrics we have analyzed, we assert that the VPI and like ratio are the best indicators of audience engagement as a viewer has to like the video while watching it. Therefore, "clickbait videos" (which may have a higher number of views due to a catchy title and thumbnail) are not part of the equation. Clickbait videos would actually have a lower VPI and like ratio score since people would dislike the video more.

\section{Context}

After deep analysis, the sources of the videos with the highest popularity were more for entertainment purposes, especially news channels or other educational channels rather than hospitals. Media has a potent influence on the public perception of any kind of illness $[26,27]$. Social media influencers should be mentioned as they can often outshine medical authorities in terms of traffic, views comments and popularity. Not to mention they profit from YouTube with ads and sponsorship deals [28]. There is no doubt that social media influencers often engage in mental health awareness as this is a popular topic among their viewers [29]. With the majority of YouTube users being between $15-25$ years old and $75 \%$ of mental health disorders being manifested before the age of 24 , it is understandable that mental health is a popular topic among this demographic [30,31].

Taking all this into consideration, it is evident that the risk of misconception is high since media coverage is bound to focus on details that create impact and interest. Therefore, it is of critical importance that health care institutions use media as a type of communication to provide accurate and high-quality information to the public. As noted previously, YouTube and other internet websites have gained a more active role in the life of patients and their management of illness or disease. During epidemics such as the recent novel corona pandemic, the role of telemedicine and online source of health information is emphasized even more. Doctors need to be aware of this and provide reliable resources for their patients. Previous YouTube quality studies found that physicians upload a relatively low number of educational or medical videos on YouTube $[3,4]$. Our study matches this finding as only $11.3 \%$ of the videos were published by physicians in our study. We encourage medical professions to use our optimization analysis findings to make their videos more appealing to the viewer.

Patients search the internet for medical information most often to learn about side effects from prescription drugs or to obtain help from coping with their disease [11]. This is especially important for patients with $\mathrm{BD}$ since the majority of them will inevitably turn to the internet for sources of health information [11]. Our study showed that videos often lacked information regarding how to cope with the disorder and what treatment they may undergo.

Patients and physicians may greatly benefit from empowering patients with unbiased and robust medical information. It has been shown to reduce diagnostic times, create online support communities and encourage a more productive patient-doctor dialogue [3,32-35].

The ability to provide reliable information at a distance is just one of the ways telemedicine is expanding. Modern methods of psychiatric patient care include E-mental health apps with medication monitoring and individualized messages based on real-time data self-reported by patients [36,37]. This is especially critical in the current era of practicing medicine at a distance. However, while these validated digital tools and helpful online resources exist, we wish to highlight the "digital divide" where people with lower technical skills or access to digital technology may not be able to utilize these resources. Indeed, a 2016 study showed that patients with low health literacy were less likely to use health information technology [38]. The current coronavirus disease 2019 pandemic has exacerbated this divide as essential services have moved online although some populations still do not have access to the internet [39].

We stress that medical information on YouTube is only supplementary and does not replace seeing a doctor. Bauer M, et al. [40] brings to attention the potential medical harm related to "poor quality online information, self-diagnosis and self-treatment, passive monitoring, and the use of unvalidated smartphone apps [40]." Moreover, there is potential to quickly share fake medical news with the use of social media. Waszak PM, et al. [41] found that popular websites related to common diseases contained misinformation in $40 \%$ of cases and were shared 451,272 times from 2012 to 2017 [41].

\section{Limitations}

One may argue that a physician with experience with $\mathrm{BD}$, such as a psychiatrist, should be involved in the video evaluation process. However, the DISCERN instrument was created for "patients and information providers" and "was not dependent on specialist knowledge of a health condition or treatment." In addition, the two raters had an excellent intraclass correlation agreement indicating the quality analysis study was reliable and conclusive. Moreover, the two independent raters were medical students both having completed 90 hours of clinical rotations in psychiatry. 
We based our main keywords on the suggestions and synonyms that the YouTube search provided. Thus, we believe that they are relevant. However, the paper would have even more robust findings if a pilot study was done to see exactly what BD patients search for online.

\section{Future directions}

Since this paper is a cross-sectional evaluation, we recommend that it be repeated within a few years to monitor alterations of video content quality. Such studies may focus on how physician and hospital-based health channels on YouTube provide medical contentbased and if it up to date with recent evidence. Additional studies may be done to examine the extent to which patients with $\mathrm{BD}$ refer to YouTube specifically to gather information about their disease and whether they regard this information as valuable. Furthermore, the YouTube algorithm that ranks and suggests videos is complex, highly personalized and constantly changing [42,43]. Moreover, new videos are being added constantly and new popular trends affect the search results (e.g., the amyotrophic lateral sclerosis ice bucket challenge of 2014). In our experience, we did not notice any popular trends concerning BD. However, we encourage future scientists to publish a follow-up study to monitor the quality of YouTube information on BD over time and obtain a clearer understanding of which video content is most frequently recommended by YouTube.

Since only English videos were analyzed future studies may focus the differences in video quality in various languages and geographical locations where there may be more stigmatization of mental diseases [44]. Furthermore, future studies could use the DISCERN instrument to evaluate other sources of medical information about mental health such as phone apps or websites. We encourage physicians to inform patients about DISCERN tool to evaluate medical health information for themselves.

\section{Conclusion}

YouTube is often a good source of information on BD as it fulfills the basic information required for patient information. However, the quality of information may still improve particularly in regards to treatment risks, consequences, benefits and overall treatment options. The unregulated nature of YouTube means that videos may fail to meet a higher standard in conveying medical information. Thus, care should be taken by health professionals to provide reliable online information. We suggest that physicians reference our list of the highest quality videos on BD. Since a doctor speaker and animation significantly optimized a video for high audience engagement, we recommend that physicians create medical content on $\mathrm{BD}$ and include animation in the video.

\section{Declaration}

\section{Ethics approval and consent to participate}

Not applicable.

\section{Consent for publication}

Not applicable.

\section{Availability of data and material}

All data was available online.

\section{Competing interests}

Not applicable.

\section{Funding}

Not applicable.

\section{Authors' contributions}

Tomasz Szmuda-Methodology, Investigation, Formal analysis, Writing-original draft

Weronika Żydowicz-Methodology, Investigation, Formal analysis, Writing-original draft

Shan Ali-Methodology, Investigation, Formal analysis, Writingoriginal draft

Karolina Fedorow-Methodology, Investigation, Formal analysis, Writing-original draft

Paweł Słoniewski-Methodology, Formal analysis, Supervision

\section{Acknowledgements}

Not applicable.

\section{Author's information}

Tomasz Szmuda, MD, PhD; Medical University of Gdansk, Neurosurgery Department

Weronika Żydowicz; Medical University of Gdansk

Shan Ali; Medical University of Gdansk

Karolina Fedorow; Medical University of Gdansk

Paweł Słoniewski MD, PhD, Professor; Medical University of Gdansk, Neurosurgery Department

\section{References}

1. Statista (2021) Global digital population as of January 2021 (in billions). Statista Inc, United States.

2. PwC Health Research Institute (2012) Social media "likes" healthcare from marketing to social business. PwC, United Kingdom.

3. Madathil KC, Rivera-Rodriguez AJ, Greenstein JS, Gramopadhye AK (2015) Healthcare information on YouTube: A systematic review. Health Informatics J 21: 173-194.

4. Szmuda T, Rosvall P, Hetzger TV, Ali S, Słoniewski P (2020) YouTube as a Source of Patient Information for Hydrocephalus: A ContentQuality and Optimization Analysis. World Neurosurg 138: e469-e477.

5. Lam NHT, Woo BKP (2020) Efficacy of Instagram in Promoting Psychoeducation in the Chinese-Speaking Population. Health Equity 4: $114-116$

6. Lam NHT, Tsiang JT, Woo BKP (2017) Exploring the Role of YouTube in Disseminating Psychoeducation. Acad Psychiatry 41: 819-822.

7. Szmuda T, Özdemir C, Fedorow K, Ali S, Słoniewski P (2021) YouTube as a source of information for narcolepsy: A content-quality and optimization analysis. J Sleep Res 30: e13053.

8. Depression and Bipolar Support Alliance (2021) Bipolar Disorder Statistics. DBSA, United States.

9. Bauer R, Conell J, Glenn T, Alda M, Ardau R, et al. (2016) Internet use by patients with bipolar disorder: Results from an international multisite survey. Psychiatry Res 242: 388-394.

10. Bauer R, Conell J, Glenn T, Alda M, Ardau R, et al. (2017) International multi-site survey on the use of online support groups in bipolar disorder. Nord J Psychiatry 71: 473-476.

11. Conell J, Bauer R, Glenn T, Alda M, Ardau R, et al. (2016) Online information seeking by patients with bipolar disorder: results from an international multisite survey. Int J Bipolar Disord 4: 17. 
12. Mileva VR, Vázquez GH, Milev R (2013) Effects, experiences, and impact of stigma on patients with bipolar disorder. Neuropsychiatr Dis Treat 9: 31-40.

13. Shen H, Zhang L, Xu C, Zhu J, Chen M, et al. (2018) Analysis of Misdiagnosis of Bipolar Disorder in An Outpatient Setting. Shanghai Arch Psychiatry 30: 93-101.

14. iProspect (2006) iProspect search engine user behaviour study. United States.

15. Charnock D, Shepperd S, Needham G, Gann R (1999) DISCERN An instrument for judging the quality of written consumer health information on treatment choices. J Epidemiol Community Health 53: 105-111.

16. Rees CE, Ford JE, Sheard CE (2002) Evaluating the reliability of DISCERN: a tool for assessing the quality of written patient information on treatment choices. Patient Educ Couns 47: 273-275.

17. Cassidy JT, Baker JF (2016) Orthopaedic Patient Information on the World Wide Web: An Essential Review. J Bone Joint Surg Am 98: 325 338.

18. Weil AG, Bojanowski MW, Jamart J, Gustin T, Lévêque M (2014) Evaluation of the quality of information on the Internet available to patients undergoing cervical spine surgery. World Neurosurg 82: e31-e39.

19. Szmuda T, Talha SM, Singh A, Ali S, Słoniewski P (2021) YouTube as a source of patient information for meningitis: A content-quality and audience engagement analysis. Clin Neurol Neurosurg 202: 106483.

20. Szmuda T, Alkhater A, Albrahim M, Alquraya E, Ali S, et al. (2020) YouTube as a source of patient information for stroke: A contentquality and an audience engagement analysis. J Stroke Cerebrovasc Dis 29: 105065

21. Szmuda T, Syed MT, Singh A, Ali S, Özdemir C, et al. (2020) YouTube as a source of patient information for Coronavirus Disease (COVID-19): A content-quality and audience engagement analysis. Rev Med Virol 30: e2132.

22. Koo TK, Li MY (2016) A Guideline of Selecting and Reporting Intraclass Correlation Coefficients for Reliability Research. J Chiropr Med 15: 155-163.

23. Terry K Koo, Mae Y Li (2017) Erratum to "A Guideline of Selecting and Reporting Intraclass Correlation Coefficients for Reliability Research." J Chiropr Med 16: 346.

24. Cicchetti DV (1994) Guidelines, Criteria, and Rules of Thumb for Evaluating Normed and Standardized Assessment Instruments in Psychology. Psychol Assess 6: 284-290.

25. Desai T, Shariff A, Dhingra V, Minhas D, Eure M, et al. (2013) Is content really king? An objective analysis of the public's response to medical videos on YouTube. PLoS One 8: 82469.

26. Young ME, Norman GR, Humphreys KR (2008) Medicine in the popular press: the influence of the media on perceptions of disease. PLoS One 3: e3552.

27. Ross AM, Morgan AJ, Jorm AF, Reavley NJ (2019) A systematic review of the impact of media reports of severe mental illness on stigma and discrimination, and interventions that aim to mitigate any adverse impact. Soc Psychiatry Psychiatr Epidemiol 54: 11-31.

28. Lindgren S, Lundström R (2019) Tube therapy: Dealing with mental health problems in social video comment threads. First Monday 24: 5-6.
29. Foster CB (2013) Mental Health On Youtube: Exploring the Potentia of Interactive Media to Change Knowledge, Attitudes and Behaviors About Mental Health. Doctoral dissertation, University of South Carolina, USA.

30. Aslam S (2020) YouTube by the Numbers: Stats, Demographics \& Fun Facts. Omnicore, United States.

31. National Alliance on Mental Illness (2021) Mental Health by the Numbers. NAMI, United States.

32. Sangeorzan I, Andriopoulou P, Livanou M (2019) Exploring the experiences of people vlogging about severe mental illness on YouTube: An interpretative phenomenological analysis. J Affect Disord 246: 422-428.

33. Nour MM, Nour MH, Tsatalou OM, Barrera A (2017) Schizophrenia on YouTube. Psychiatr Serv 68: 70-74.

34. Michalak EE, McBride S, Barnes SJ, Wood CS, Khatri N, et al. (2017) Bipolar disorder research 2.0: Web technologies for research capacity and knowledge translation. J Eval Clin Pract 23: 1144-1152.

35. Naslund JA, Grande SW, Aschbrenner KA, Elwyn G (2014) Naturally occurring peer support through social media: the experiences of individuals with severe mental illness using YouTube. PLoS One 9: e110171.

36. Faurholt-Jepsen M, Frost M, Christensen EM, Bardram JE, Vinberg $M$, et al. (2020) Validity and characteristics of patient-evaluated adherence to medication via smartphones in patients with bipolar disorder: exploratory reanalyses on pooled data from the MONARCA I and II trials. Evid Based Ment Health 23: 2-7.

37. Faurholt-Jepsen $\mathrm{M}$, Frost $\mathrm{M}$, Christensen EM, Bardram JE, Vinberg $\mathrm{M}$ et al. (2019) The validity of daily patient-reported anxiety measured using smartphones and the association with stress, quality of life and functioning in patients with bipolar disorder. J Affect Disord 257: 100-107.

38. Mackert M, Mabry-Flynn A, Champlin S, Donovan EE, Pounders K (2016) Health Literacy and Health Information Technology Adoption: The Potential for a New Digital Divide. J Med Internet Res 18: e264.

39. Lai J, Widmar NO (2021) Revisiting the Digital Divide in the COVID-19 Era. Appl Econ Perspect Policy

40. Bauer M, Glenn T, Monteith S, Bauer R, Whybrow PC, et al. (2017) Ethical perspectives on recommending digital technology for patients with mental illness. Int J Bipolar Disord 5: 6

41. Waszak PM, Kasprzycka-Waszak W, Kubanek A (2018) The spread of medical fake news in social media-The pilot quantitative study. Heal Policy Technol 7: 115-118.

42. Covington P, Adams J, Sargin E (2016) Deep neural networks for YouTube recommendations. RecSys '16: Proceedings of the $10^{\text {th }}$ ACM Conference on Recommender Systems, New York, USA 191198.

43. Davidson J, Liebald B, Liu J, Nandy P, Van Vleet T (2010) The YouTube video recommendation system. RecSys'10: Proceedings of the fourth ACM conference on Recommender systems 293-296.

44. Botha UA, Koen L, Niehaus DJ (2006) Perceptions of a South African schizophrenia population with regards to community attitudes towards their illness. Soc Psychiatry Psychiatr Epidemiol 41: 619 623. 